\title{
Synchronization in MIMO OFDM systems
}

\author{
L. Häring and A. Czylwik \\ Department of Communication Systems, University of Duisburg-Essen, Bismarckstr. 81, 47057 Duisburg, Germany
}

\begin{abstract}
In this paper, an overview of carrier frequency offset (CFO) estimation algorithms for Orthogonal Frequency Division Multiplexing (OFDM) systems is presented. It is well-known that multicarrier systems suffer from their high sensitivity to mismatches of transmitter and receiver oscillator frequencies. The performance degrades since the CFO destroys the orthogonality of the subcarriers. Hence, extensive research has been done on the estimation and correc-

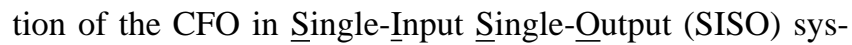
tems. Mainly, the proposed algorithms can be categorized into data-aided and blind techniques. Several estimation techniques have been extended to the Single-Input MultipleOutput (SIMO) case where multiple receive antennas can be utilized to gain diversity. However, less attention has been paid on synchronization in the attractive Multiple-Input Multiple-Output (MIMO) case which is topic of tremendous interest in current research. The present paper concentrates on aspects of this new scenario. Starting with algorithms for SISO and SIMO, this contribution reviews briefly proposed carrier frequency synchronization techniques which could be implemented in forthcoming MIMO systems.
\end{abstract}

\section{Introduction}

In recent years, OFDM has become increasingly popular for future high data-rate wireless communication systems because of its advantages in a multipath environment. It has been standardized for Digital Audio Broadcasting (DAB), Terrestrial Digital Video Broadcasting (DVB-T) as well as for broadband wireless local area networks (WLAN) i.e. Hiperlan/2 and IEEE 802.11a/g. However, one of the main drawbacks is its high sensitivity to frequency offsets since the orthogonality between subcarriers is destroyed thus leading to a considerable system performance degradation (Pollet et al., 1995). Hence, the estimation and correction of fre- quency offsets have been subject of intensive research in the last decade. But it has not been investigated extensively in systems with multiple antennas at transmitter and receiver site. Recently, such MIMO systems have become attractive since the capacity was proven (under ideal conditions) to scale with the minimum number of transmit and receive antennas (Foschini and Gans, 1998). Therefore, the contribution introduces thoroughly a MIMO system model that considers CFOs. Furthermore, it gives an overview of important existing estimation algorithms for SISO systems and extensions to multiple antenna systems.

The remainder of the paper is organized as follows. Section 2 presents the OFDM system model for the SISO and MIMO case as well as impacts of frequency offsets on the system performance. In Sect. 3, important classes of SISO algorithms are reviewed. Extensions of these algorithms to multiple antenna systems are discussed in Sect. 4. To illustrate some important aspects, Sect. 5 presents a brief simulation example. Finally, in Sect. 6 conclusions are drawn.

\section{OFDM signal model}

We start with the OFDM model formulation for the SISO case. Subsequently, we extend the model to a MIMO system (see Fig. 1).

\subsection{System model in SISO}

In OFDM, information data are transmitted blockwise. A sequence of complex data symbols is split into blocks and fed to different subcarriers. For the $k$-th block, an IDFT operation on the symbols of all carriers is carried out, which can be expressed as

$$
x_{i}(k)=\frac{1}{\sqrt{N}} \sum_{n=0}^{N_{\mathrm{u}}-1} s_{n}(k) \mathrm{e}^{\mathrm{j} \frac{2 \pi}{N} n i}, \quad 0 \leq i \leq N-1,
$$



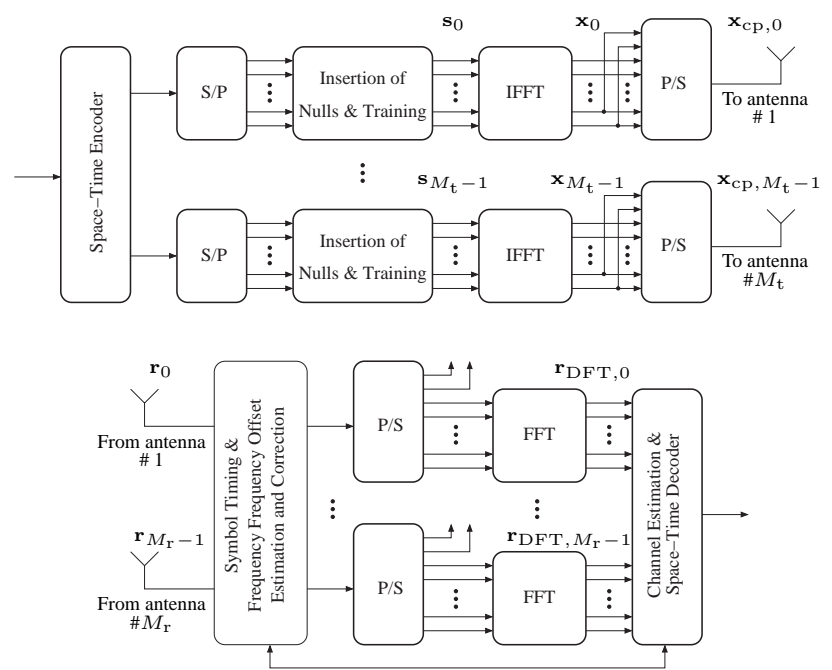

Fig. 1. Block diagram of transmitter and receiver in a MIMO OFDM system.

where $N, N_{\mathrm{u}}(\leq N)$ and $s_{n}(k)$ denote the IDFT-size, the number of used subcarriers and data symbols of the $n$-th subcarrier, respectively. If $N_{\mathrm{u}}<N$, the residual $N-N_{\mathrm{u}}$ subcarriers referred to as null or virtual subcarriers are filled with zeros. With the defined vectors

$\begin{aligned} \mathbf{s}(k) & =\left[s_{0}(k), s_{1}(k), \ldots, s_{N_{\mathrm{u}}-1}(k)\right]^{T} \\ \mathbf{x}(k) & =\left[x_{0}(k), x_{1}(k), \ldots, x_{N_{\mathrm{u}}-1}(k), \ldots, x_{N-1}(k)\right]^{T}\end{aligned}$

and the IDFT-matrix

$\mathbf{W}_{N_{\mathrm{u}}}=\frac{1}{\sqrt{N}}\left(\begin{array}{cccc}1 & 1 & \ldots & 1 \\ 1 & \mathrm{e}^{\mathrm{j} \frac{2 \pi}{N}} & \ldots & \mathrm{e}^{\mathrm{j} \frac{2 \pi}{N}\left(N_{\mathrm{u}}-1\right)} \\ \vdots & \vdots & \ddots & \vdots \\ 1 \mathrm{e}^{\mathrm{j} \frac{2 \pi}{N}(N-1)} & \ldots & \mathrm{e}^{\mathrm{j} \frac{2 \pi}{N}(N-1)\left(N_{\mathrm{u}}-1\right)}\end{array}\right)$,

the $k$-th block can be written in a more compact way:

$\mathbf{x}(k)=\mathbf{W}_{N_{\mathrm{u}}} \mathbf{s}(k)$.

Subsequently, a cyclic prefix of length $G$ is preceded, which is just a duplication of the last $G$ samples:

$\mathbf{x}_{\mathrm{cp}}(k)=[\underbrace{x_{N-G}(k), \ldots, x_{N-1}(k)}_{\text {cyclic prefix }}, x_{0}(k), \ldots, x_{N-1}(k)]^{T}$.

In order to avoid interblock interference (IBI), $G$ must be chosen larger than the length $L$ of the channel impulse response. After passing through a pulse-shaping filter with the impulse response $g_{\mathrm{t}}(t)$ (e.g. root-raised cosine), the transmitted time-continuous waveform yields

$x_{\mathrm{t}}(t)=\sum_{k=-\infty}^{\infty} \sum_{i=-G}^{N-1} x_{\mathrm{cp}, i+G}(k) g_{\mathrm{t}}\left(t-k N_{\mathrm{b}} T-i T\right)$,

where $T$ is the sampling period and $N_{\mathrm{b}}=N+G$ denotes the length of one OFDM block.
At the receiver, mismatches between transmitter and receiver oscillators as well as Doppler effects result in a frequency offset $f_{\mathrm{o}}$. Usually, the most dominant frequency offset arises from oscillator imperfections.

Assuming a frequency-selective channel with the timeinvariant impulse response $h_{\mathrm{c}}(t)$ and a receiver filter with $g_{\mathrm{r}}(t)$ which is matched to the transmit filter, we can write for the effective channel impulse response $h(t)=g_{\mathrm{t}}(t) * h_{\mathrm{c}}(t) *$ $g_{\mathrm{r}}(t)$. The received time-continuous waveform containing a frequency offset yields then

$r(t)=\mathrm{e}^{\mathrm{j}\left(2 \pi f_{\mathrm{o}} t+\phi\right)} \sum_{k=-\infty}^{\infty} \sum_{i=-G}^{N-1} x_{\mathrm{cp}, i+G}(k) h\left(t-k N_{\mathrm{b}} T-i T\right)$,

where noise contributions are omitted and $\phi$ is an arbitrary phase. Without loss of generality, we set $\phi=0$.

Sampled at time instants $t=k^{\prime} N_{\mathrm{b}} T+i^{\prime} T^{1}$ and using the normalized offset $\varepsilon=f_{\mathrm{o}} / \Delta f$ related to the carrier subspacing $\Delta f=1 /(N T)$, one obtains with Eq. (1)

$$
\begin{aligned}
r_{i^{\prime}}\left(k^{\prime}\right)= & \mathrm{e}^{\mathrm{j} \frac{2 \pi}{N} \varepsilon\left(k^{\prime} N_{\mathrm{b}}+i^{\prime}\right)} \frac{1}{\sqrt{N}} \sum_{k=-\infty}^{\infty} \sum_{i=-G}^{N-1} \sum_{n=0}^{N_{\mathrm{u}}-1} s_{n}(k) . \\
& \mathrm{e}^{\mathrm{j} \frac{2 \pi}{N} n i} h\left(\left(k^{\prime}-k\right) N_{\mathrm{b}} T+\left(i^{\prime}-i\right) T\right) .
\end{aligned}
$$

Furthermore, we assume that the duration of the combined channel impulse response is limited such that $h(t)=$ $0 \forall t \backslash[0, L T]$, where $L<G$. Hence, the only nonzero contribution in Eq. (5) comes from $k=k^{\prime}$ :

$r_{i^{\prime}}\left(k^{\prime}\right)=\mathrm{e}^{\mathrm{j} \frac{2 \pi}{N} \varepsilon\left(k^{\prime} N_{\mathrm{b}}+i^{\prime}\right)} \frac{1}{\sqrt{N}} \sum_{n=0}^{N_{\mathrm{u}}-1} s_{n}\left(k^{\prime}\right) \sum_{i=i^{\prime}}^{i^{\prime}-L} h\left(i^{\prime}-i\right) \mathrm{e}^{\mathrm{j} \frac{2 \pi}{N} n i}$.

The sampling period $T$ was omitted for notational convenience. Using the combined channel transfer function $H_{n}=$ $\sum_{i=0}^{L} h(i) \mathrm{e}^{-\mathrm{j} \frac{2 \pi}{N} n i}$, the $k^{\prime}$-th received OFDM-block for $i^{\prime} \in$ $[0, \ldots, N-1]$ after removal of the cyclic prefix is given by

$$
\begin{aligned}
r_{i^{\prime}}\left(k^{\prime}\right)= & \mathrm{e}^{\mathrm{j} \frac{2 \pi}{N} \varepsilon\left(k^{\prime} N_{\mathrm{b}}+i^{\prime}\right)} \frac{1}{\sqrt{N}} \sum_{n=0}^{N_{\mathrm{u}}-1} s_{n}\left(k^{\prime}\right) . \\
& \underbrace{\sum_{i^{\prime}-i=0}^{L} h\left(i^{\prime}-i\right) \mathrm{e}^{-\mathrm{j} \frac{2 \pi}{N} n\left(i^{\prime}-i\right)}}_{H_{n}} \mathrm{e}^{\mathrm{j} \frac{2 \pi}{N} n i^{\prime}} \\
= & \mathrm{e}^{\mathrm{j} \frac{2 \pi}{N} \varepsilon\left(k^{\prime} N_{\mathrm{b}}+i^{\prime}\right)} \cdot \operatorname{IDFT}_{i^{\prime}}\left\{s_{n}\left(k^{\prime}\right) H_{n}\right\} .
\end{aligned}
$$

Alternatively, in matrix formulation if $k^{\prime}$ is substituted by $k$ and a block fading channel is assumed, we obtain

$\mathbf{r}(k)=\mathrm{e}^{\mathrm{j} \frac{2 \pi}{N} \varepsilon k N_{\mathrm{b}}} \mathbf{C}_{\varepsilon} \mathbf{W}_{N_{\mathrm{u}}} \mathbf{H}(k) \mathbf{s}(k)$

\footnotetext{
${ }^{1}$ For reasons of simplicity and space limitation, in this paper, perfect timing synchronization is assumed. Timing synchronization in OFDM systems involves finding the optimal position of the DFTwindow for demodulation. Due to margins between the length of guard interval and channel impulse response in practical systems, requirements with respect to timing synchronization are relaxed.
} 


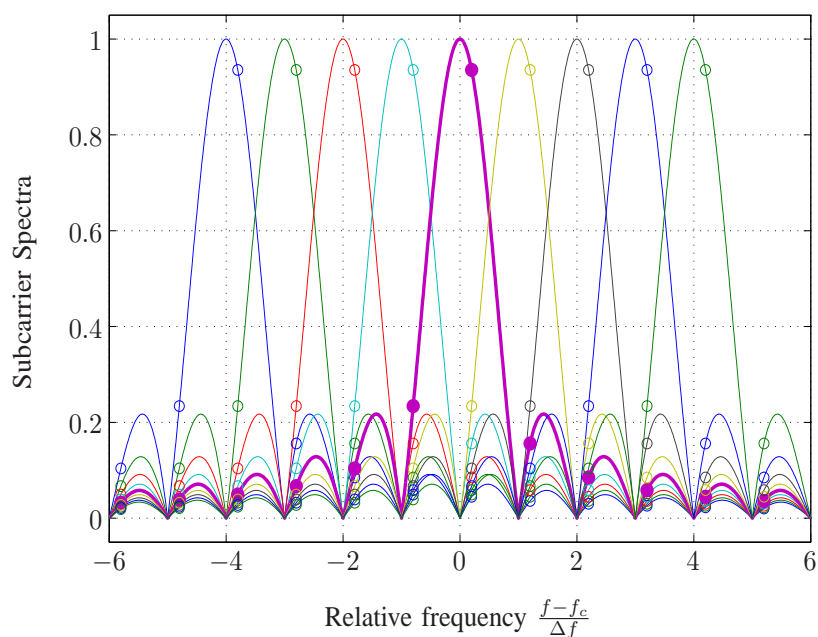

Fig. 2. Effects of a CFO $(\varepsilon=0.2)$.

with $\mathbf{C}_{\varepsilon}=\operatorname{diag}\left(1, \mathrm{e}^{\mathrm{j} \frac{2 \pi}{N} \varepsilon}, \ldots, \mathrm{e}^{\mathrm{j} \frac{2 \pi}{N} \varepsilon(N-1)}\right)$ denoting the diagonal $N \times N$ carrier frequency offset matrix and $\mathbf{H}(k)=\operatorname{diag}\left(H_{0}(k), \ldots, H_{N_{\mathrm{u}}-1}(k)\right)$ representing the diagonal $N_{\mathrm{u}} \times N_{\mathrm{u}}$ channel matrix. A CFO turns out to rotate the phase of the received samples linearly with time. To demodulate, we left-multiply $\mathbf{r}(k)$ with the DFT matrix $\mathbf{W}_{N_{\mathrm{u}}}^{H}$ :

$\mathbf{r}_{\mathrm{DFT}}(k)=\mathrm{e}^{\mathrm{j} \frac{2 \pi}{N} \varepsilon k N_{\mathrm{b}}} \mathbf{W}_{N_{\mathrm{u}}}^{H} \mathbf{C}_{\varepsilon} \mathbf{W}_{N_{\mathrm{u}}} \mathbf{H}(k) \mathbf{s}(k)$.

If the matrix $\mathbf{W}_{N_{\mathrm{u}}}^{H} \mathbf{C}_{\varepsilon} \mathbf{W}_{N_{\mathrm{u}}}$ is non-diagonal, the orthogonality between subcarriers is lost, thus resulting in inter-carrier interference (ICI). To analyze this further and using some straightforward derivation, we can find

$\mathbf{r}_{\mathrm{DFT}}(k)=\mathrm{e}^{\mathrm{j} \frac{2 \pi}{N} \varepsilon k N_{\mathrm{b}}} \mathbf{B H}(k) \mathbf{s}(k)$

with

$\mathbf{B}=\left(\begin{array}{ccc}b_{0} & \ldots & b_{N_{\mathrm{u}}-1} \\ \vdots & \ddots & \vdots \\ b_{-N_{\mathrm{u}}+1} & \ldots & b_{0}\end{array}\right)$

where

$b_{n}=\frac{\sin (\pi(\varepsilon+n))}{N \sin \left(\frac{\pi}{N}(\varepsilon+n)\right)} \mathrm{e}^{\mathrm{j} \pi \frac{N-1}{N}(\varepsilon+n) .}$

Equations (9)-(11) indicate two effects of CFOs: The amplitudes of the desired subcarriers multiplied by $b_{0}$ are reduced and cross terms (for $n \neq 0$ ) are introduced thus confirming the above mentioned statement. Figure 2 illustrates both issues.

In his work (Moose, 1994), Moose investigated the impact of a CFO analytically. He calculated a lower bound of the signal-to-interference-and-noise power ratio (SINR) visualized in Fig. 3 for different signal-to-noise power ratios (SNR). It can be clearly seen, that especially at high SNR regions, the performance degradation caused by synchronization errors becomes considerable.

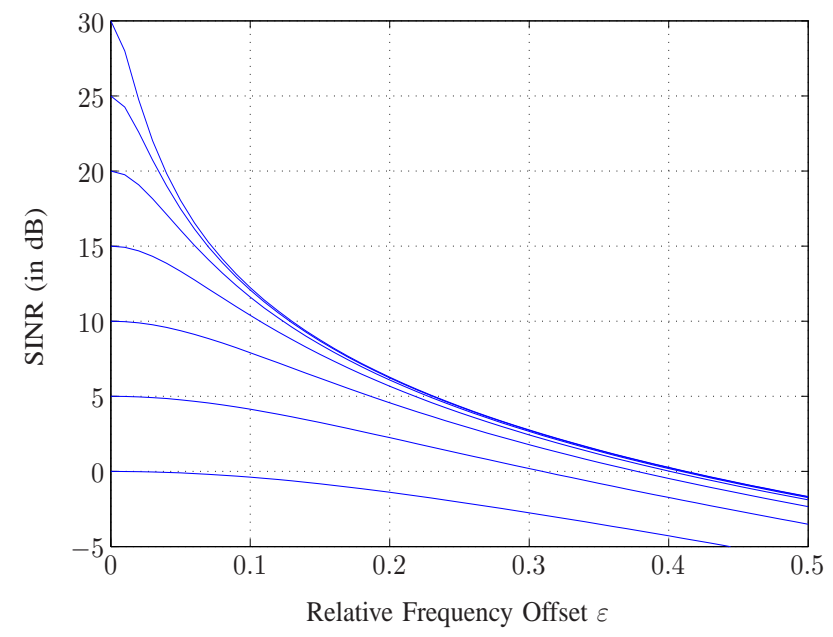

Fig. 3. Lower Bound of SINR (Moose, 1994).

\subsection{Extension to MIMO}

In the following, it is considered that $M_{\mathrm{t}}$ transmit and $M_{\mathrm{r}}$ receive branches share the same oscillator, respectively. With this practical assumption, still only one CFO emerges. Hence, the $k$-th signal block at the $m_{\mathrm{r}}$-th receive antenna after cyclic prefix removal can be written as

$\mathbf{r}_{m_{\mathrm{r}}}(k)=\mathrm{e}^{\mathrm{j} \frac{2 \pi}{N} \varepsilon k N_{\mathrm{b}}} \mathbf{C}_{\varepsilon} \mathbf{W}_{N_{\mathrm{u}}} \sum_{m_{\mathrm{t}}=0}^{M_{\mathrm{t}}-1} \mathbf{H}_{m_{\mathrm{r}}, m_{\mathrm{t}}}(k) \mathbf{s}_{m_{\mathrm{t}}}(k)$,

where $\mathbf{s}_{m_{\mathrm{t}}}(k)$ denotes the block vector transmitted from the $m_{\mathrm{t}}$-th transmit antenna. $\mathbf{H}_{m_{\mathrm{r}}, m_{\mathrm{t}}}(k)$ is a diagonal $N_{\mathrm{u}} \times N_{\mathrm{u}}$ matrix describing the frequency channel characteristic from the $m_{\mathrm{t}}$-th transmit antenna to the $m_{\mathrm{r}}$-th receive antenna. Stacking blocks from all antennas together,

$\begin{aligned} \tilde{\mathbf{s}}(k) & =\operatorname{vec}\left(\mathbf{s}_{0}(k), \ldots, \mathbf{s}_{M_{\mathrm{t}}-1}(k)\right) \\ \tilde{\mathbf{r}}(k) & =\operatorname{vec}\left(\mathbf{r}_{0}(k), \ldots, \mathbf{r}_{M_{\mathrm{r}}-1}(k)\right),\end{aligned}$

we can find the following compact notation:

$\tilde{\mathbf{r}}(k)=\mathrm{e}^{\mathrm{j} \frac{2 \pi}{N} \varepsilon k N_{\mathrm{b}}} \tilde{\mathbf{C}}_{\varepsilon} \tilde{\mathbf{W}}_{N_{\mathrm{u}}} \operatorname{diag}\left(\mathbf{H}_{0}(k), \ldots, \mathbf{H}_{M_{\mathrm{r}}-1}(k)\right) \breve{\mathbf{s}}(k)$

$\tilde{\mathbf{H}}(k)$

with $M_{\mathrm{r}} M_{\mathrm{t}} N_{\mathrm{u}} \times 1$ vector $\breve{\mathbf{s}}(k)=\operatorname{vec}(\tilde{\mathbf{s}}(k), \ldots, \tilde{\mathbf{s}}(k))$ and

$$
\begin{aligned}
\tilde{\mathbf{C}}_{\varepsilon} & =\mathbf{I}_{M_{\mathrm{r}}} \otimes \mathbf{C}_{\varepsilon} \\
\tilde{\mathbf{W}}_{N_{\mathrm{u}}} & =\mathbf{I}_{M_{\mathrm{r}}} \otimes \mathbf{W}_{N_{\mathrm{u}}} \\
\mathbf{H}_{m_{\mathrm{r}}}(k) & =\left[\mathbf{H}_{m_{\mathrm{r}}, 0}(k), \ldots, \mathbf{H}_{m_{\mathrm{r}}, M_{\mathrm{t}}-1}(k)\right] .
\end{aligned}
$$

Here, $\otimes$ denotes the Kronecker product and $\mathbf{I}_{K}$ the $K \times K$ identity matrix. Taking the DFT by left-multiplication $\tilde{\mathbf{r}}(k)$ with $\tilde{\mathbf{W}}_{N_{\mathrm{u}}}^{H}=\mathbf{I}_{M_{\mathrm{r}}} \otimes \mathbf{W}_{N_{\mathrm{u}}}^{H}$ yields

$$
\begin{aligned}
\tilde{\mathbf{r}}_{\mathrm{DFT}}(k) & =\mathrm{e}^{\mathrm{j} \frac{2 \pi}{N} \varepsilon k N_{\mathrm{b}}} \tilde{\mathbf{W}}_{N_{\mathrm{u}}}^{H} \tilde{\mathbf{C}}_{\varepsilon} \tilde{\mathbf{W}}_{N_{\mathrm{u}}} \tilde{\mathbf{H}}(k) \breve{\mathbf{s}}(k) \\
& =\mathrm{e}^{\mathrm{j} \frac{2 \pi}{N} \varepsilon k N_{\mathrm{b}}}\left(\mathbf{I}_{M_{\mathrm{r}}} \otimes \mathbf{B}\right) \tilde{\mathbf{H}}(k) \breve{\mathbf{s}}(k) .
\end{aligned}
$$




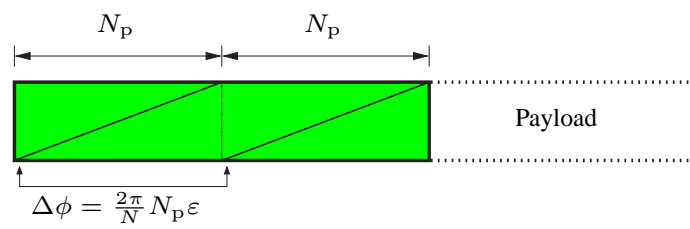

Fig. 4. Preamble structure consisting of two identical halves (Moose, 1994).

It can be seen that the influence of a CFO is the same for all receive antennas. Similarly to Eq. (8), to restore the orthogonality between subcarriers, the unknown parameter $\varepsilon$ in $\widetilde{\mathbf{C}}_{\varepsilon}$ needs to be estimated, preferably before carrying out the DFT. The estimate $\hat{\varepsilon}$ can be used to derotate the received samples before demodulation.

Algorithms that find an estimate $\hat{\varepsilon}$ can be mainly classified in data-aided and blind methods (dependent on the information that is available). Although also possible in the frequency-domain (after DFT processing) (e.g. Santella, 2000 , and references therein), this paper only deals with methods working on time-domain samples.

\section{Frequency synchronization in SISO systems}

\subsection{Data-aided approaches}

In many data-aided synchronization approaches, algorithms are based upon the observation (e.g. in Eq. 6), that CFOs only cause linear (related to time) phase shifts of the received symbols. Hence, frequency offsets can be estimated by "measuring" the phase rotation (unless the phase rotation does not exceed an angle of $2 \pi$ ). To follow this approach, Moose proposed in Moose (1994) two identical training symbols as a preamble (see Fig. 4).

From one symbol to the other, the phase changes by $\Delta \phi=$ $\frac{2 \pi}{N} N_{\mathrm{p}} \varepsilon$ :

$r_{i+N_{\mathrm{p}}}=r_{i} \mathrm{e}^{\mathrm{j} \frac{2 \pi}{N} N_{\mathrm{p}} \varepsilon}+n_{i}, \quad i=0, \ldots, N_{\mathrm{p}}-1$,

where $N_{\mathrm{p}}$ denotes the length of one training symbol. In the original work, Moose proposed $N_{\mathrm{p}}=N$ und used samples in the frequency domain. For $n_{i}$ representing additive white Gaussian noise (AWGN), he calculated the MaximumLikelihood (ML) estimator:

$\hat{\varepsilon}=\frac{1}{2 \pi} \frac{N}{N_{\mathrm{p}}} \angle R=\frac{1}{2 \pi} \frac{N}{N_{\mathrm{p}}} \angle\left(\sum_{i=0}^{N_{\mathrm{p}}-1} r_{i}^{*} r_{i+N_{\mathrm{p}}}\right)$,

where $\angle R$ denotes the angle of the complex correlation sum.

The fact that some timing synchronization techniques rely upon computing the correlation sum in Eq. (19), argues for the practical usefulness of this algorithm. Furthermore, although mathematically shown only for an AWGN channel, the estimator in Eq. (19) turns out to perform well even in frequency-selective fading channels since the periodicity of

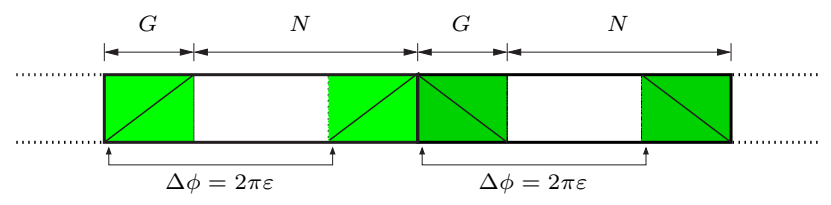

Fig. 5. OFDM block structure with cyclic prefix.

the training structure is still preserved (if a cyclic prefix is preceded).

However, one major drawback of this method is its short estimation range of $|\varepsilon|<\frac{N}{2 N_{\mathrm{p}}}$ caused by phase ambiguities. Shortening the length of the training symbols increases the acquisition range, but lowers the estimation performance as well. Several researchers have investigated possibilities to extend the range by modifying the preamble - however, mainly by cost of some increase in computational load. Moreover, two-step procedures with two different training sequences consisting of a course and fine estimation step have also been proposed as well as symbols with more than two identical halves. The interested reader is referred to e.g. Schmidl and Cox (1997), Morelli and Mengali (1999), Li et al. (2001).

\subsection{Blind approaches}

In van de Beek et al. (1997), VAN DE BEEK et al. proposed to exploit the cyclic prefix which is essential anyway to prevent IBI and furthermore helps to simplify the channel equalization. Originally, the authors found an optimal joint estimator for both timing and frequency synchronization. With the correlation between received symbols of the cyclic prefix and of its duplicate for the $k$-th block (signal power $\sigma_{s}^{2}$ )

$\mathrm{E}\left\{r_{i}^{*}(k) r_{i+N}(k)\right\}=\sigma_{s}^{2} \mathrm{e}^{\mathrm{j} 2 \pi \varepsilon}, \quad i=0, \ldots, G-1$,

the ML estimate of the CFO in an AWGN channel yields

$\hat{\varepsilon}=\frac{1}{2 \pi} \angle R(k)=\frac{1}{2 \pi} \angle\left(\sum_{i=0}^{G-1} r_{i}^{*}(k) r_{i+N}(k)\right)$.

In order to enhance the estimation performance, averaging over subsequent OFDM blocks is beneficial. Besides the fixed small estimation range of half of the carrier spacing, the estimator in Eq. (21) - in contrast to the data-aided approach - suffers from considerable performance degradation in frequency-selective fading channels. At high SNR, it exhibits a large error floor. Recently proposed methods try to adapt VAN DE BEEK's approach to multipath channels. The idea is to use statistical properties of the received signal to weight contributions in the correlation sum dependent on how heavily they are corrupted by IBI.

Another important class of blind estimation methods utilizes redundancy introduced by virtual subcarriers. These carriers can be regarded as spectral guard regions that are often used in practical OFDM systems to relax requirements of 
the analog radio frequency (RF) front-end. Additionally, a major advantage of these algorithms is the larger acquisition range.

The basic idea was found by LiU and Tureli (Liu and Tureli, 1998) first: Let us assume $N-N_{\mathrm{u}}$ virtual carriers, thus the "full" IDFT-matrix becomes $\mathbf{W}=$ $\left[\mathbf{W}_{N_{\mathrm{u}}}, \mathbf{w}_{N_{\mathrm{u}}+1}, \ldots, \mathbf{w}_{N}\right]$.

Due to the orthogonality property of column vectors of $\mathbf{W}$, we obtain in absence of CFO and noise $\left(n=1, \ldots, N-N_{\mathrm{u}}\right)$

$\mathbf{w}_{N_{\mathrm{u}}+n}^{H} \mathbf{r}(k)=\mathbf{w}_{N_{\mathrm{u}}+n}^{H} \mathbf{W}_{N_{\mathrm{u}}} \mathbf{H}(k) \mathbf{s}(k)=0$.

This basic observation leads to the following problem formulation to estimate $\varepsilon$ :

$$
\begin{aligned}
J(e) & =\sum_{k=0}^{K-1} \sum_{n=1}^{N-N_{\mathrm{u}}}\left|\mathbf{w}_{N_{\mathrm{u}}+n}^{H} \mathbf{C}_{e}^{-1} \mathbf{r}(k)\right|^{2} \\
\hat{\varepsilon} & =\arg \min _{e} J(e) .
\end{aligned}
$$

The estimator in Eq. (23), similar to the (in array processing) well-known MUSIC algorithm, is equivalent to the nonlinear least squares (NLS) solution. Under some uncritical assumptions, the cost function in Eq. (23) is shown to yield a unique minimum. However, MA et al. have proven in Ma et al. (2001) the loss of identifiability in case of "channel nulls". To guarantee channel-independent identiafibility, hopping virtual subcarriers (positions of these carriers change from block to block) have been suggested.

Yet another blind synchronization algorithms proposed by BÖLCSKEI that also has got a large acquisition range is based upon the second-order statistics of OFDM signals. BöLCSKEI has shown in Bölcskei (2001) that cyclostationarity is introduced by a cyclic prefix, pulse shaping or by the use of different transmit powers on the subcarriers such that the second-order statistics contain information on the synchronization parameters.

The interested reader is referred to Bölcskei (2001) and Gini and Giannakis (1998).

\section{Frequency synchronization in SIMO and MIMO sys- tems}

As stated before, if transmitter and receiver branches share a common oscillator, respectively (which was considered throughout this paper), evidently still only one frequency offset in Eq. (14) must be estimated. Thus in principle, all the described algorithms can be also used for such a MIMO scenario. However, spatial diversity can be exploited in order to enhance the estimator performance.

\subsection{Data-aided approaches}

Unfortunately, for the synchronization unit, channel state information is not available which complicates any diversity approach. This general problem was investigated by CZYLWIK in Czylwik (1999) for SIMO systems; the synchronization algorithm used in his paper was based upon MoosE's

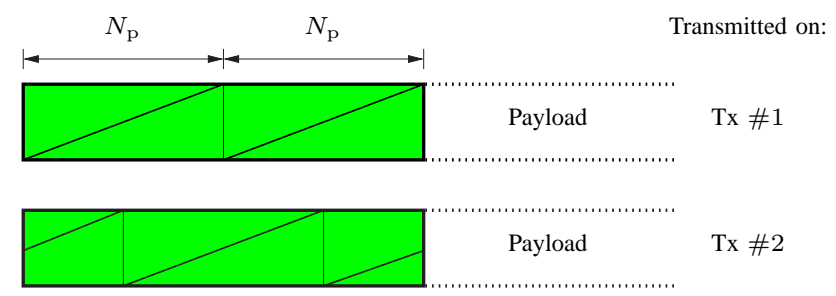

Fig. 6. Modified preamble suitable for MIMO transmission (Schenk and van Zelst, 2003).

approach. He found out that the optimum of any linear combination of uncorrelated received signals leads to a selection combing (SC) concept, where only the strongest branch signal passes. However, if a non-linear operation is performed first, also information from other antennas can be advantageously utilized by a method similar to maximum ratio combining (MRC), in which branch weights are set in order to maximize the SNR.

A synchronization unit for MIMO systems based upon MOOSE's and VAN DE BEEK's approach was suggested by Mody et al. (Mody and Stüber, 2001). But diversity concepts have not been sufficiently explored in this proposal. The main novelty was the modification of training sequences. Generally, the same training sequences as for synchronization are also used for channel estimation. Hence, additional requirements have to be met. The authors suggested the usage of chirp like orthogonal sequences proposed by Suehiro (Suehiro and Hatori, 1998).

Recently in 2003, SCHENK et al. (Schenk and van Zelst, 2003) analyzed a MIMO extension of Moose (1994) also from an analytical point of view. In this approach, they used constant-envelope orthogonal codes with good periodic correlation properties, such as FRANK-ZADOFF codes (Frank and Zadoff, 1962). Training sequences on different antennas are cyclically shifted as illustrated in Fig. 6 for $M_{\mathrm{t}}=2$.

Instead of averaging estimates on different antenna signals which is equivalent to equal gain combining (EGC), the estimator is calculated

$\hat{\varepsilon}=\frac{1}{2 \pi} \frac{N}{N_{\mathrm{p}}} \angle \tilde{R}=\frac{1}{2 \pi} \frac{N}{N_{\mathrm{p}}} \angle\left(\sum_{m_{\mathrm{r}}=0}^{M_{\mathrm{r}}-1} R_{m_{\mathrm{r}}}\right)$,

where $R_{m_{\mathrm{r}}}$ denotes the complex correlation sum of each antenna branch. $R_{m_{\mathrm{r}}}$ is composed in the same way as in Eq. (19). In a frequency-selective fading channel, the estimator yields a MRC-like performance since antenna signals with a higher SNR contribute more to the estimate.

For an AWGN channel, the Cramer-Rao bound (CRB) is Schenk and van Zelst (2003)

$\operatorname{var} \hat{\varepsilon} \geq \frac{N}{(2 \pi)^{2} \cdot N_{\mathrm{p}}^{3} \cdot M_{\mathrm{r}} \cdot \mathrm{SNR}}$.

Clearly, the CRB depends on the number of receive antennas rather than on the number of transmit antennas. We will see later, however, that in a fading channel also transmit diversity can enhance the estimator performance. 


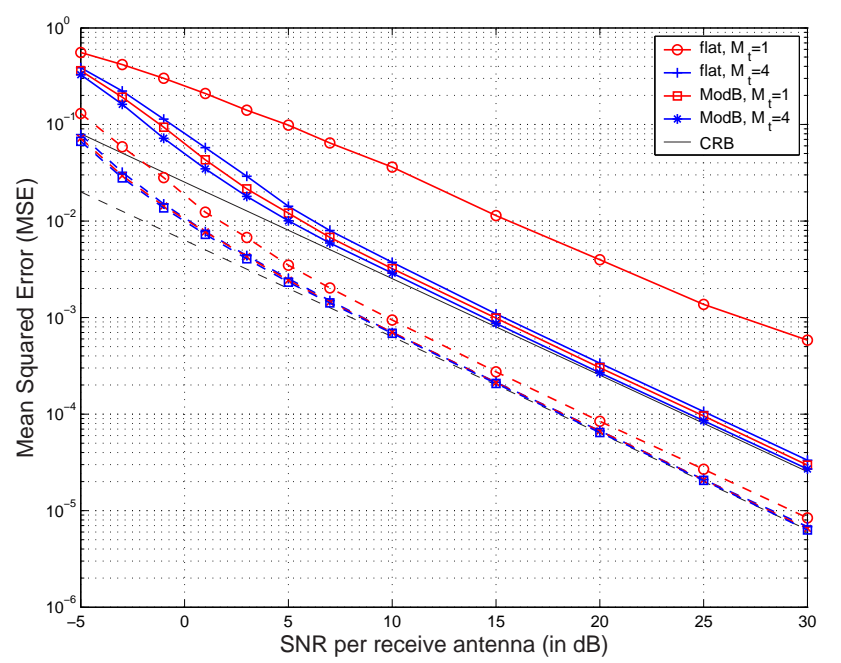

Fig. 7. CRB and simulated MSE of the CFO estimation for different combinations of $M_{\mathrm{t}}$ and $M_{\mathrm{r}}=1$ (solid, -) and $M_{\mathrm{r}}=4$ (dashed, --) averaged over 20000 realizations.

\subsection{Blind approaches}

Again motivated by Czylwik (1999), HonAN et al. also extended the blind estimation algorithm based on virtual subcarriers to the SIMO case (e.g. in Honan et al., 2003). Path gains $\left|\gamma_{m_{\mathrm{r}}}\right|$ are introduced in the cost function:

$$
\begin{aligned}
J(e) & =\sum_{k=0}^{K-1} \sum_{m_{\mathrm{r}}=0}^{M_{\mathrm{r}}-1} \sum_{n=1}^{N-N_{\mathrm{u}}}\left|\gamma_{m_{\mathrm{r}}}\right|^{2}\left|\mathbf{w}_{N_{\mathrm{u}}+n}^{H} \mathbf{C}_{e}^{-1} \mathbf{r}_{m_{\mathrm{r}}}(k)\right|^{2} \\
\hat{\varepsilon} & =\arg \min _{e} J(e) .
\end{aligned}
$$

The authors proved that the weights $\left|\gamma_{m_{\mathrm{r}}}\right|$ facilitate estimator MRC diversity gain when selected proportional to the branch SNR. They validated also in an experimental setup the superior performance of this approach compared to EGC especially at low SNRs. At higher SNR, EGC and MRC coincide.

The cost function in Eq. (26) can also be used in MIMO scenarios. In Honan and Tureli (2003) it is shown that the CRB in AWGN channels does not change with the number of transmit antennas. However, the frequency offset estimator benefits from transmit diversity which leads to an essential performance improvement at lower SNR. In addition, the MIMO diversity gain helps the estimator to be less sensitive in case of channel nulls.

\section{Simulation example}

Due to space limitation, only a short simulation example of the MIMO estimation algorithm proposed by SCHENK is following to demonstrate the main aspects.

To have a fair comparison, the total power radiated from $M_{\mathrm{t}}$ transmit antennas in MIMO is restricted to $\sigma_{s}^{2}$, which means that the transmitted power per antenna is reduced by the factor $M_{\mathrm{t}}$. Simulations have been carried out for an
AWGN and frequency-selective fading channel. The multipath channel is implemented as a tapped-delay line with Rayleigh fading coefficients and a power delay profile given by typical indoor models used for WLANs at $5 \mathrm{GHz}$ (Medbo and Schramm, 1998). Impinging signals at different antennas are assumed to be uncorrelated. We choose the DFT-size $N=64$, the sampling period $T=50 \mathrm{~ns}$ (e.g. in IEEE 802.11 a) and periodicity length $N_{\mathrm{p}}=16$. As a cyclic prefix, one period of 16 samples is preceded. The relative CFO error in all simulations is $\varepsilon=0.2$. To study the influence of multiple antennas, the mean squared error (MSE) of $\hat{\varepsilon}$ versus SNR per receive antenna for a different number of $M_{\mathrm{t}}$ and $M_{\mathrm{r}}$ is simulated.

In Fig. 7, the simulation results for both a flat and frequency-selective (Model B for typical large open space and office environment (Medbo and Schramm, 1998), average rms delay spread of $100 \mathrm{~ns}$ ) fading channel are depicted. For reference, also the CRB for AWGN is displayed.

As expected, we can observe a performance degradation in fading channels compared to the CRB (as well as for a simulated AWGN channel which is not displayed here), especially at low SNRs. (At high SNRs, apart from the SISO flat fading case, the MSE approximates the CRB, which reveals a ML estimator.) The degradation decreases with increasing delay spread due to the gain of frequency diversity. It is interesting to note that the estimator does not suffer from multipath since the periodicity of training symbols is preserved.

However, performance improvement is also achieved by space diversity. The effect of transmit diversity becomes apparent for $M_{\mathrm{t}}=4$ versus $M_{\mathrm{t}}=1$ in a flat fading channel using only a single receive antenna. Nevertheless, receive diversity plays the dominant role since for $M_{\mathrm{r}}>1$ the estimator is less sensitive to fading and furthermore lowers the bound of the MSE. This simulation example shows that frequency offset estimation in MIMO systems is more robust under different fading conditions, which can be explained by the space diversity introduced by multiple antennas.

\section{Conclusion}

A MIMO OFDM system model including a carrier frequency offset has been developed and the effects of such offsets on the system performance in OFDM systems have been illustrated. Basic SISO data-aided and blind estimation algorithms using time-domain samples have been reviewed. Moreover, some extensions to a SIMO and MIMO scenario that efficiently exploit spatial diversity have been presented. Finally, a simulation example has been shown to demonstrate the main aspects. 


\section{References}

Bölcskei, H.: Blind estimation of symbol timing and carrier frequency offset in wireless OFDM systems, IEEE Trans. Comm., 49, 6, 988-999, 2001.

Czylwik, A.: Synchronization for systems with antenna diversity, Proc. of IEEE Veh. Technol. Conf., 2, 728-732, 1999.

Foschini, G. and Gans, M.: On limits of wireless communications in a fading environment when using multiple antennas, Wireless Personal Communications, 6, 311-335, 1998.

Frank, R. and Zadoff, S.: Phase Shift Pulse Codes with good periodic correlation properties, IRE Trans. on Inform. Theory, IT-8, 381-382, 1962.

Gini, F. and Giannakis, G. B.: Frequency offset and symbol timing recovery in Flat-Fading channels: A cyclostationary approach, IEEE Trans. Comm., 46, 400-411, 1998.

Honan, P. J. and Tureli, U.: Blind Carrier Offset Estimation for MIMO OFDM systems: Cramer Rao Bound and Nonlinear Least Squares, in Proc. of Conf. on Inform. Sciences and Systems, 2003.

Honan, P. J., Ambati, R., and Tureli, U.: Performance Analysis of Diversity Combining Method for OFDM Blind Carrier Synchronization, Proc. of IEEE Veh. Technol. Conf., 4, 2672-2676, 2003.

Li, J., Liu, G. Q., and Giannakis, G. B.: Carrier frequency offset estimation for OFDM-based WLANs, IEEE Signal Proc. Letters, 8, 80-82, 2001

Liu, H. and Tureli, U.: A high-efficiency carrier estimator for OFDM commucations, IEEE Commun. Letters, 2, 104-106, 1998.

Ma, X. L., Tepedelenlioglu, C., Giannakis, G. B., and Barbarossa, S.: Non-Data-Aided Carrier offset estimators for OFDM with null subcarriers: Identifiability, algorithms, and performance, IEEE J. Select. Areas Commun., 19, 2504-2515, 2001.
Medbo, J. and Schramm, P.: Channel Models for HIPERLAN/2 in Different Indoor Scenarios, ETSI/BRAN document no. 3ERI085B, 1998.

Mody, A. N. and Stüber, G. L.: Synchronization for MIMO systems, Proc. of IEEE Globecom Conf., 1, 509-513, Texas, USA, 2001.

Moose, P. M.: A technique for Orthogonal Frequency Division Multiplex frequency offset correction, IEEE Trans. Comm., 42, 1994.

Morelli, M. and Mengali, U.: An improved frequency offset estimator for OFDM applications, IEEE Comm. Letters, 3, 75-77, 1999.

Pollet, T., van Bladel, M., and Moeneclaey, M.: BER sensitivity of OFDM systems to carrier frequency offset and Wiener phase noise, IEEE Trans. Comm., 43, 191-193, 1995.

Santella, G.: A Frequency and Symbol Synchronization System for OFDM Signals: Architecture and Simulation Results, IEEE Trans. Veh. Technol., 49, 254-275, 2000.

Schenk, T. and van Zelst, A.: Frequency Sychronization for MIMO OFDM wireless LAN systems, in Proc. of IEEE Veh. Technol. Conf., 509-513, Florida, USA, 2003.

Schmidl, T. and Cox, D.: Robust frequency and timing synchronization for OFDM, IEEE Trans. Comm., 45, 1613-1621, 1997.

Suehiro, N. and Hatori, M.: Modulatable Orthogonal Sequences and their Application to SSMA Systems, Trans. on Inform. Theory, 34, 93-100, 1998.

van de Beek, J., Sandell, M., and Börjesson, P.: ML estimation of time and frequency offset in OFDM systems, IEEE Trans. Signal Proc., 45, 1800-1805, 1997. 